\title{
How we are born
}

\author{
Sudhansu K. Dey
}

Division of Reproductive Sciences, The Perinatal Institute, Cincinnati Children's Hospital Medical Center, University of Cincinnati College of Medicine, Ohio.

\begin{abstract}
Human reproduction is relatively inefficient. Nearly $30 \%$ of pregnancies result in spontaneous losses, which are both a clinical problem and a psychological stress to the families involved. Furthermore, although the human population is growing rapidly and is predicted to reach 9 billion by $2050,15 \%$ of couples worldwide are childless because of infertility. Many underlying causes of infertility have been overcome by assisted reproductive technologies such as in vitro fertilization, yet pregnancy success rates using such approaches remain disappointingly low. Since mechanistic approaches to study human reproductive processes are ethically restricted, future advances in fertility treatment and the development of new contraceptives rely predominantly on the study of the factors influencing reproduction in model systems. The articles in this Reproductive Biology Review series present updates on the current understanding of various reproductive processes in model systems and raise questions that need to be addressed if we are to improve human reproductive health.
\end{abstract}

Viviparity, meaning giving birth to live offspring that have developed within the maternal body, is a momentous stage in evolution. The most evolutionarily advanced form of viviparity is placental viviparity, in which the embryo derives continuous nourishment from the mother via a placenta. It is a form of reproduction found in most mammals and a few other species, including scorpions and some sharks and snakes. The more primitive form of viviparity, ovoviviparity, refers to embryo development within an egg that is retained within the maternal body; in this case, the embryo derives food from the yolk present in the egg.

In placental mammals (eutherians), sexual reproduction occurs through highly complex and orchestrated processes that successfully propagate and diversify genetic information to progeny. Such procreation begins with germ cell development and ends with the birth of live young. A well-controlled succession of events involving germ cell differentiation, gametogenesis, ovulation, fertilization, preimplantation embryo development, implantation, decidualization (differentiation of uterine stromal cells into specialized cells, termed decidual cells, at the site of implanting embryo), placentation, and parturition directs the birth of live offspring (Figure 1). The success of each event is contingent upon the success of the preceding event, and dysregulation of any of the events along this pathway preclude a live birth. Carrying an embryo in utero to live birth is therefore an enduring task for the mother and necessitates many safeguards throughout the course of pregnancy.

The embarkment of a new life begins with the union of a haploid sperm and a haploid egg, leading to fertilization and the formation of a diploid one-cell zygote. The one-cell zygote undergoes several mitotic cell divisions, forming a differentiated embryo at the blastocyst stage. Blastocysts have two distinct cell populations: one forms the inner cell mass (ICM), and the other the trophectoderm (TE) layer that surrounds the ICM (1). At the time of implantation, the ICM further differentiates into two distinct cell lineages, the epiblast (EPI) and primitive endoderm (PE). Whereas the EPI gives rise to the fetus proper, the PE and TE take extraembryonic fates as the fetal membranes and placenta, respectively. A reciprocal interaction between the blastocyst and the maternal uterus initiates the process of implantation, which results in the vascular system of the embryo being connected to the maternal circulation, leading to the forma-

Conflict of interest: The author has declared that no conflict of interest exists. Citation for this article: J Clin Invest. 2010;120(4):952-955. doi:10.1172/JCI42380. tion of a functional placenta and the establishment of pregnancy. The process of parturition completes the course of pregnancy and results in the birth of an offspring. More detailed basic understanding of how these events are coordinated is crucial if we are to move from bench to bedside, enhancing pregnancy success rates in fertility clinics and developing new strategies for novel contraceptives. To this end, it is hoped that the articles in this Review series, which comprise an overview of our current understanding of many of the events crucial to sexual reproduction in placental mammals, point some of the ways forward to achieving these goals.

\section{Sexual reproduction: an update}

In this collection of Reviews, the authors present studies of model systems to summarize current understanding of the genetic and molecular pathways that direct various reproductive events. In addition, they highlight many of the questions that need to be answered if progress is to be made in improving human reproductive health.

From a male perspective. In the first Review in the series (2), Cathryn A. Hogarth and Michael D. Griswold describe the process of spermatogenesis, focusing on what is known in the mouse, as well as the intricate interactions that occur between germ cells and the somatic cells that support their development. These interactions, modulated by the combined actions of follicle-stimulating hormone (FSH), testosterone, and retinoic acid (vitamin A), regulate the three phases of spermatogenesis from mitosis of undifferentiated progenitor cells (spermatogonia), to meiosis, to differentiation of haploid cells into spermatozoa. Spermatogenesis in the seminiferous tubule is highly organized and moves along the tubule as a wave, with groups of germ cells at different stages of the differentiation pathway placed along the entire length of the tubule; the result of this is continual asynchronous generation of spermatozoa. Hogarth and Griswold rightfully state that although a wealth of information has been forthcoming from rodent models, much needs to be learned about causes of male reproductive deficiencies in humans (2). For example, vitamin A deficiency leads to many human diseases, including night blindness (3), but not much is known about the effects of its deficiency on human reproductive performance. Although environmental estrogens and endocrine disrupters (exogenous substances that behave like hormones and interfere with the body's endocrine system) are linked to declining male reproductive performance in humans and other animals, more mechanistic information needs to be obtained 


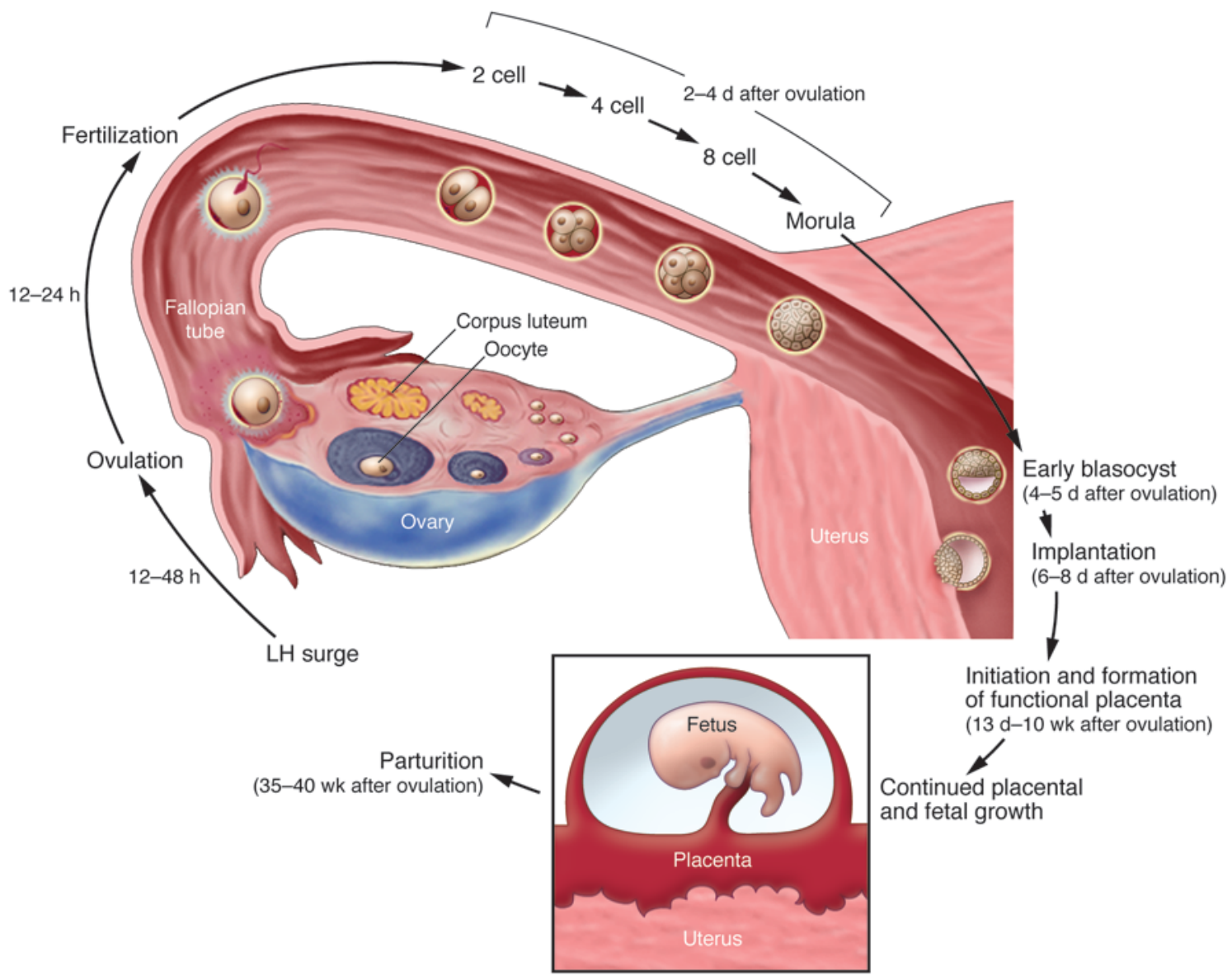

Figure 1

Timeline of pregnancy events in humans. This timeline is an approximation based on a 28- to 30-day menstrual cycle, the average length of a woman's menstrual cycle. A surge in luteinizing hormone (LH) levels occurs at approximately day 13 of the menstrual cycle, and this stimulates ovulation. The ruptured follicle forms a corpus luteum, which produces large amounts of progesterone that modify the uterus lining so that it is receptive to implantation of an embryo if fertilization has occurred. Fertilization takes place in the fallopian tube. As the fertilized egg travels down toward the uterus, it undergoes a series of early cleavage divisions, producing increasing numbers of progressively smaller cells, known as blastomeres, without changing the overall size of the embryo. The 16-cell-stage embryo is known as a morula, and its cells are indistinguishable. As further cleavage steps occur and the blastocoel develops, the morula becomes a blastocyst. The blastocyst implants into the uterine lining approximately 6-8 days after ovulation. A fully functional placenta does not form until approximately 10 weeks after ovulation. Parturition at full term occurs 40 weeks after ovulation.

about their sites of action, the downstream signaling pathway involved, and the duration and extent of their exposure in affecting the male reproductive system.

From a female perspective. In the second Review (4), JoAnne S. Richards and Stephanie A. Pangas describe basic ovarian biology and its clinical implications. Undifferentiated, proliferating primordial germ cells (PGCs) migrate from the embryonic yolk sac and colonize the indifferent gonad (the term for a gonad in early embryonic life, as the gonads do not have male or female identity, an identity that comes later in development when the indifferent gonads differentiate into testes in males and ovaries in females). Richards and Pangas discuss how morphogens, growth factors, and transcription factors direct indifferent gonads to form ovaries rather than testes in mice (4). The plasticity of indifferent gonads such that various environmental and hormonal cues can direct them to a specific genotype-phenotype is remarkable and is seen throughout the animal kingdom, but it raises interesting questions as to the significance of this plasticity: Is it a function of gene-environment interactions? Does it serve as an evolutionary watchdog to balance the male and female sex ratio under environmental and population density pressures? Is it more vulnerable to sex specification error? These questions warrant further investigation.

PGCs in the ovary differentiate to form oocytes. The ovarian reserve of oocytes is drastically reduced prenatally in humans and shortly after birth in mice. Richards and Pangas describe the various roles of TGF- $\beta$ family members and Notch signaling in primordial follicle formation and oocyte maturation (4). They also highlight how gonadotropins and various growth factors, transcription factors, and signaling pathways orchestrate follicle development and ovulation. While they caution that phytoestrogens (naturally occurring nonsteroidal plant compounds that are structurally similar to estrogens) and environmental estrogens might have adverse effects on follicular development, they also propose that preserving the size of the primordial follicle pool may extend 
the life of ovarian function, compromising the effects of diseases associated with menopause, such as osteoporosis, cardiovascular disease, and gynecological cancers. One possible way to preserve the ovarian follicle reserve would be to prevent the loss of follicles that undergo premature demise - a process known as follicular atresia - before ovulation in each cycle. Why and how follicular atresia occurs still remains unanswered.

In their Review (5), Roger Gosden and Bora Lee underline the complex bidirectional interactions between oocytes and their follicular microenvironment that govern oocyte maturation and follicular development. Oocyte development cannot be realized without the participation of a follicle that provides the suitable microenvironment for the oocyte to become competent to produce a viable embryo after fertilization. Although granulosa cells within follicles provide an essential niche for nourishment and development of the oocyte, factors emanating from the oocyte also regulate granulosa cell function. This mutual interaction between the two entities confers normal synchronized development of the follicle and the oocyte; failure of this synchrony results in compromised follicle development and oocyte maturation.

Gosden and Lee point out that oocyte quality is a major determinant for the success of assisted reproductive technologies (ARTs) such as in vitro fertilization (IVF) (5). They underscore the crucial roles of oocyte cytoplasm in the maturation process that involves epigenetic alterations and translation of stored mRNAs during transcriptional silence, which prevails from the time of meiotic resumption through the early stages of embryo cleavage. The authors stress the need for further research efforts on cytoplasmic maturation to improve oocyte quality and developmental competence, examining the successes and shortcomings of ARTs in the context of oocyte quality as well as alternative methods of deriving oocytes through stem cell and/or cloning technologies. An in-depth understanding of the preservation of primordial follicles, and later their culture in vitro to generate mature follicles and oocytes, may help cancer survivors after undergoing chemotherapy and/or radiotherapy or women past their normal reproductive age to have children.

Meeting of the sperm and egg. Moving forward in the events that lead to sexual reproduction, Masaru Okabe and colleagues discuss the various steps that ensure fertilization, that is, successful fusion of a sperm with an egg (6). The various genetic and molecular pathways that affect sperm migration and capacitation (maturation of sperm to be competent for fertilizing eggs) in the female reproductive tract, leading to sperm-cumulus interaction, are discussed. The mechanisms underlying the acrosome reaction (a reaction that occurs in the acrosome, a cap-like structure situated in the sperm head, that enables the sperm to fuse with and penetrate through the zona pellucida of the egg), binding to the zona pellucida (the thick glycoprotein shell that protects growing oocytes), penetration of the zona pellucida, and fusion of the sperm and egg are also highlighted. Okabe and colleagues point out that many factors previously thought crucial at specific stages in the course of fertilization have been found to be nonessential based on studies in knockout mice. Studies in gene-deleted mice, however, have resulted in the identification of many new critical factors that orchestrate various events in the fertilization pathway, including cation channel, sperm associated (CATSPER; ref. 7) and izumo sperm-egg fusion (IZUMO; ref. 8), and the number of new factors is growing with the increasing availability of these mutant mice.

However, it still remains unclear whether and how sperm movement through the reproductive tract is coordinated by its intrinsic motility and the surrounding microenvironment guidance cues. The discovery of sperm capacitation independently by Min Chueh Chang and Colin Russell Austin six decades ago $(9,10)$ led to the successful development of IVF techniques and ultimately the birth of the first test tube baby. Advancements in understanding of spermatogenesis, sperm biology, and fertilization research have been skillfully used to improve the success of ARTs, resulting in soaring use of clinical practices performing these techniques, but have not yet been fully exploited to develop male contraception.

After sperm-egg union. Fertilization in the female reproductive tract is the initiation of the process of making a blastocyst for implantation in the uterus. In their Review (11), Katie Cockburn and Janet Rossant discuss how a fertilized egg blossoms into a differentiated blastocyst with two distinct initial cell populations, the ICM and TE, and how the ICM further differentiates into the EPI and PE. They explain how the dependence of the zygote on maternal mRNA for protein synthesis is transitioned to zygotic genome activation (ZGA) during the early embryonic cleavage stages and point out differences in this event between mice and humans. Then they walk us through a series of events that encompasses compaction (flattening of the embryonic cells at the morula stage of development such that the cell outlines are indistinguishable), cell polarity, symmetric versus asymmetric cell division, and blastocoel formation (a fluid-filled cavity within the embryo essential for formation of the three embryonic germ layers), all of which are essential to blastocyst formation. Various cellular, molecular, and signaling pathways associated with these processes are also described. Cockburn and Rossant then describe the stage-specific totipotency and developmental plasticity of preimplantation embryos in vivo and in vitro, elucidating their significance in stem cell biology and ART. Although the discussion is mostly mouse centric, they insightfully point out the critical differences between mouse and human preimplantation embryo development. Because the quality of preimplantation embryos greatly affects pregnancy outcome in humans, continued research to illuminate the cellular and molecular landscape of preimplantation biology will help improve the quality and rate of pregnancy. Attention also needs to be directed toward improving embryo culture conditions, minimizing the impact of environmental stress and epigenetic alterations, in order to produce embryos of superior quality for IVF.

The final journey. Dynamic and intimate interactions between the implantation-competent blastocyst and the receptive uterus are essential for successful implantation and homing of the embryo within the uterine cavity. Any aberration of implantation or deferral of timely implantation creates an adverse ripple effect throughout the subsequent course of pregnancy, compromising pregnancy outcome (12). H. Jade Lim and Haibin Wang discuss in their Review (13) various phases of uterine sensitivity to implantation and subsequent decidualization, primarily citing studies in mice, but pointing out their relevance to humans. The authors outline the genetic and molecular pathways that prepare the uterus to become receptive to implantation, facilitate the attachment of the blastocyst to the uterine lumen, and initiate stromal cell proliferation and differentiation to decidual cells (i.e., decidualization). One interesting difference between rodents and humans is that whereas decidualization in rodents is triggered by blastocyst attachment to the uterine lining, it occurs in women in the absence of an embryo; however, this process is further accentuated by blastocyst implantation. Although the significance of this predecidualization event is not clearly understood, it is perhaps similar to the 
extensive stromal cell proliferation that occurs in mice on day 4 of pregnancy, prior to implantation, under the influence of ovarian estrogen and progesterone (12).

Lim and Wang also discuss in detail how the master regulators estrogen and progesterone control the stage-specific participation in preparing the uterus for implantation of spatiotemporally expressed morphogens, growth factors, cytokines, transcription factors, lipid mediators, cell cycle regulators, and endocannabinoids. These studies are discussed in the context of developing a better understanding of human uterine disorders such as ectopic pregnancy, endometriosis, intrauterine growth retardation, preeclampsia, and preterm labor.

How the uterus prepares for implantation presents many challenging questions. Although a few signaling pathways are known to be critical for uterine receptivity, almost nothing is known at the molecular and genetic level about how the uterus spontaneously transitions from a receptive to a nonreceptive phase. Thus, there is a continuing need to unravel the complexities of uterine receptivity and nonreceptivity to address two contrasting global issues: infertility and a lack of novel contraceptives.

The final major event during pregnancy is the formation of a functional placenta. Maternal resources, filtered across the selective barrier that is the placenta, nourish and protect the conceptus. Placental function is terminated with the completion of parturition. In the final Review of this series (14), Emin Maltepe, Anna I. Bakardjiev, and Susan J. Fisher shed light on the formation of the placenta, which, in addition to filtering maternal resources, also fulfills hormonal and transport functions between maternal and fetal circulations. The authors highlight a number of developmental processes, such as angiogenesis, branching morphogenesis, trophoblast differentiation (generation of the extraembryonic epithelial cells - trophoblasts - that form the placenta), and syncytium formation (a multinucleated population of cells crucial for placental function) that contribute to the formation of this functional selective transport system. Maltepe and colleagues also emphasize the importance of the coordinated interactions of various transcription factors and epigenetic regulators with the microenvironment in regulating stem cell differentiation pathways crucial to formation of the mature placenta. They believe that a better understanding of these pathways would improve in vitro culture conditions for trophoblast stem cell-based experiments, the results of which would help better define placental pathologies that contribute to intrauterine fetal growth restriction, preterm delivery, and hypertensive disorders of preeclampsia. The authors rightfully point out that thorough comprehension of placental biology is of great importance, because the quality of life of a growing fetus in utero is likely to be a major determinant of quality of life in adulthood, as noted by Barker in 1994 (15). David Barker's hypothesis underscores the concept that fetal distress in utero heralds the adult onset of diseases. This has been highlighted again in a recent article indicating that intrauterine fetal exposure to DNA damage leads to premature aging in adult life (16). The underlying mechanism by which fetal programming in utero affects adult life requires intense research using various model systems. Questions also remain unanswered regarding the role of the deciduum and/or the embryo in governing placentation, and research focus should be directed toward illuminating the cellular and molecular crosstalk among the embryo, deciduum, and placenta in order to fully appreciate the functions of these tissues in pregnancy maintenance and outcome.

\section{What the future holds}

Although the articles in this Review series provide updates on various aspects of reproductive biology, many fundamental issues remain unaddressed by the field. For example, information on the roles of short and long noncoding RNAs in various reproductive events is still limited compared with information on their functions in other organ systems and pathologic conditions. Future research must strive to answer some of the questions posited by the authors of this series and investigate other emerging research frontiers in order to improve reproductive health in humans. In this respect, creation of mouse models with regulatable conditional loss- and gainof-function of genes at specific sites and pregnancy stages of interest will be valuable to better understand human pregnancy events.

Address correspondence to: Sudhansu K. Dey, Cincinnati Children's Research Foundation, Division of Reproductive Sciences, MLC 7045, 3333 Burnet Avenue, Cincinnati, OH 45229-3039. Phone: 513.803.1158; Fax: 513.803.1160; E-mail: sk.dey@cchmc.org.
1. Gardner RL, Papaioannou VE. Differentiation in the trophectoderm and inner cell mass. In: Balls $\mathrm{M}$, Wild AE, eds. The early development of mammals. London, United Kingdom: Cambridge University Press. 1975: 107-132.

2. Hogarth CA, Griswold MD. The key role of vitamin A in spermatogenesis. JClin Invest. 2010;120(4):956-962.

3. West KP Jr. Vitamin A deficiency disorders in children and women. Food Nutr Bull. 2003; 24(4 Suppl.):S78-S90.

4. Richards JS, Pangas SA. The ovary: basic biology and clinical implications. J Clin Invest. 2010; 120(4):963-972.

5. Gosden R, Lee B. Portrait of an oocyte: our obscure origin. J Clin Invest. 2010;120(4):973-983.

6. Ikawa M, Inoue N, Benham AM, Okabe M. Fertil- ization: a sperm's journey to and interaction with the oocyte. J Clin Invest. 2010;120(4):984-994.

7. Ren D, et al. A sperm ion channel required for sperm motility and male fertility. Nature. 2001; 413(6856):603-609.

8. Inoue $\mathrm{N}$, Ikawa $\mathrm{M}$, Isotani $\mathrm{A}$, Okabe $\mathrm{M}$. The immunoglobulin superfamily protein Izumo is required for sperm to fuse with eggs. Nature. 2005; 434(7030):234-238.

9. Chang MC. Fertilizing capacity of spermatozoa deposited into the fallopian tubes. Nature. 1951; 168(4277):697-698.

10. Austin CR. Observations on the penetration of the sperm in the mammalian egg. Aust J Sci Res B. 1951; 4(4):581-596.

11. Cockburn K, Rossant J. Making the blastocyst: lessons from the mouse. JClin Invest. 2010;120(4):995-1003.

12. Wang H, Dey SK. Roadmap to embryo implantation: clues from mouse models. Nat Rev Genet. 2006; 7(3):185-199.

13. Lim HJ, Wang H. Uterine disorders and pregnancy complications: insights from mouse models. J Clin Invest. 2010;120(4):1004-1015.

14. Maltepe E, Bakardjiev AI, Fisher SJ. The placenta: transcriptional, epigenetic, and physiological integration during development. J Clin Invest. 2010; 120(4):1016-1025.

15. Barker DJ. The Wellcome Foundation Lecture, 1994. The fetal origins of adult disease. Proc Biol Sci. 1995;262(1363):37-43.

16. Fernandez-Capetillo $O$. Intrauterine programming of ageing. EMBO Reports. 2010;11(1):32-36. 